Supporting information for:

\title{
The structure and interactions of human apolipoprotein C-II in dodecyl phosphocholine
}

Christopher A. MacRaild, Geoffrey J. Howlett, and Paul R. Gooley

Russell Grimwade School of Biochemistry and Molecular Biology, University of

Melbourne, Parkville, Victoria, 3010, AUSTRALIA.

Table S1: Chemical shifts of assigned resonances in apoC-II

Figure S1: Strips, corresponding to residues 13-21, of the ${ }^{15 N-T O C S Y-H S Q C ~(b l a c k ~}$ contours) and the HNHB (red contours). Assignments of backbone and sidechain peaks are labelled. Each strip has a width of $0.15 \mathrm{ppm}$ in ${ }^{1} \mathrm{H}$ and shows a single ${ }^{15} \mathrm{~N}$ plane, centred at the chemical shifts shown. 
Table S1: Chemical shifts (ppm) of assigned resonances in apoC-II

\begin{tabular}{|c|c|c|c|c|c|}
\hline Residue & $\mathrm{N}$ & $\mathrm{HN}$ & $\mathrm{H} \alpha$ & $\mathrm{H} \beta$ & Others \\
\hline $\mathrm{T} 13$ & 113.48 & 8.27 & 4.45 & 4.64 & H $\gamma 1.37$ \\
\hline F14 & 123.44 & 8.86 & 4.49 & 3.17 & \\
\hline L15 & 118.27 & 8.50 & 3.92 & 1.64 & $\mathrm{H} \gamma(1.83 ; \mathrm{H} \delta 1.02$ \\
\hline T16 & 115.76 & 7.83 & 3.91 & 4.36 & $\mathrm{H} \gamma 1.33$ \\
\hline Q17 & 121.32 & 8.00 & 4.17 & 2.15 & \\
\hline V18 & 120.71 & 8.24 & 3.59 & 2.13 & $\mathrm{H} \gamma 0.90,0.72$ \\
\hline K19 & 119.94 & 8.24 & 3.83 & 1.98 & \\
\hline E20 & 118.18 & 8.34 & 4.17 & 2.21 & H $\gamma 2.59$ \\
\hline S21 & 116.69 & 8.23 & 4.45 & $4.10,3.96$ & \\
\hline L22 & 120.69 & 8.37 & 4.39 & 1.68 & Н $\gamma$ 1.97; H $\delta 0.94$ \\
\hline S23 & 113.86 & 8.26 & 4.28 & 4.15 & \\
\hline S24 & 116.65 & 8.15 & 4.40 & 3.94 & \\
\hline Y25 & 120.93 & 8.14 & 4.54 & 3.18 & \\
\hline W26 & 121.41 & 8.39 & 4.68 & 2.74 & \\
\hline E27 & 116.88 & 8.50 & 3.86 & 2.20 & \\
\hline S28 & 115.86 & 8.11 & 4.30 & $4.00,4.09$ & \\
\hline A29 & 111.07 & 8.54 & 4.06 & 1.50 & \\
\hline K30 & 117.62 & 8.20 & 3.59 & 1.42 & \\
\hline T31 & 115.55 & 7.86 & 3.98 & & $\mathrm{H} \gamma 1.31$ \\
\hline A32 & 124.56 & 8.08 & 4.25 & 1.56 & \\
\hline A33 & 120.09 & 8.50 & 4.05 & 1.54 & \\
\hline Q34 & 117.04 & 8.16 & 4.01 & 2.25 & $\mathrm{H} \gamma 2.55$ \\
\hline N35 & 117.56 & 8.15 & 4.59 & $2.99,2.87$ & \\
\hline L36 & 120.14 & 8.15 & 4.21 & 1.70 & $\mathrm{H} \gamma 1.90 ; \mathrm{H} \delta 0.96$ \\
\hline Y37 & 119.84 & 8.40 & 4.23 & 3.28 & \\
\hline E38 & 116.78 & 8.08 & 4.08 & 2.20 & \\
\hline K39 & 117.14 & 7.84 & 4.38 & 1.98 & \\
\hline $\mathrm{T} 40$ & 113.12 & 7.68 & 4.21 & 1.18 & \\
\hline Y41 & 121.41 & 7.93 & 4.64 & $3.12,2.99$ & \\
\hline L42 & 124.03 & 8.24 & 4.47 & & \\
\hline \multicolumn{6}{|l|}{ P43 } \\
\hline A44 & 119.26 & 7.98 & 4.37 & 1.58 & \\
\hline V45 & 118.56 & 7.82 & 3.81 & 2.36 & $\mathrm{H} \gamma 1.09,0.99$ \\
\hline D46 & 118.95 & 8.29 & 4.04 & 2.00 & \\
\hline E47 & 118.29 & 8.24 & 4.04 & 2.18 & \\
\hline K48 & 119.39 & 7.85 & 4.33 & 2.04 & \\
\hline L49 & 119.58 & 8.45 & 4.16 & & \\
\hline R50 & 122.39 & 8.33 & 4.82 & 2.08 & Н $\gamma 2.69 ; \mathrm{H} \delta 4.69$ \\
\hline D51 & 121.00 & 8.18 & 4.57 & 2.91 & \\
\hline L52 & 120.29 & 8.34 & 4.16 & 1.72 & \\
\hline Y53 & 120.79 & 8.71 & 4.20 & $3.32,3.18$ & \\
\hline S54 & 115.90 & 8.42 & 4.16 & & \\
\hline K55 & 120.65 & 8.12 & 4.33 & 1.98 & \\
\hline
\end{tabular}




\begin{tabular}{llllll} 
S56 & 115.98 & 8.09 & 4.36 & 3.96 & \\
T57 & 114.61 & 8.03 & 4.09 & 4.26 & $\mathrm{H} \gamma 1.14$ \\
A58 & 124.99 & 7.98 & 4.27 & 1.53 & \\
A59 & 120.99 & 8.02 & 4.37 & 1.57 & \\
M60 & 116.93 & 8.21 & 4.41 & 2.23 & \\
S61 & 114.64 & 8.16 & 4.41 & 4.07 & \\
T62 & 115.72 & 7.90 & 4.22 & 4.15 & $\mathrm{H} \gamma 1.09$ \\
Y63 & 120.52 & 8.03 & 4.61 & $3.25,3.05$ & \\
T64 & 112.67 & 8.07 & 4.19 & 4.38 & $\mathrm{H} \gamma 1.34$ \\
G65 & 110.91 & 8.26 & 4.03 & & \\
I66 & 119.82 & 7.79 & 4.15 & & \\
F67 & 120.21 & 8.24 & 4.53 & 3.26 & \\
T68 & 113.50 & 8.23 & 4.01 & 4.46 & $\mathrm{H} \gamma 1.35$ \\
D69 & 120.55 & 8.44 & 4.49 & 2.84 & \\
Q70 & 119.05 & 8.21 & 4.23 & 2.22 & $\mathrm{H} \gamma 2.48$ \\
V71 & 119.58 & 7.95 & 3.68 & 2.18 & $\mathrm{H} \gamma 0.94$ \\
L72 & 118.65 & 8.30 & 4.06 & 1.64 & \\
S73 & 113.89 & 7.96 & 4.23 & 4.06 & \\
V74 & 120.88 & 7.64 & 3.99 & 2.33 & $\mathrm{H} \gamma 1.17,1.04$ \\
L75 & 119.35 & 7.92 & 4.17 & & \\
K76 & 116.77 & 8.00 & 4.22 & 1.94 & \\
G77 & 122.54 & 7.98 & 4.06 & & \\
E78 & 119.52 & 8.11 & 4.45 & $2.25,2.02$ & $\mathrm{H} \gamma 2.42$ \\
E79 & 124.70 & 7.87 & 4.22 & $2.15,2.01$ & $\mathrm{H} \gamma 2.41$ \\
\hline \hline
\end{tabular}




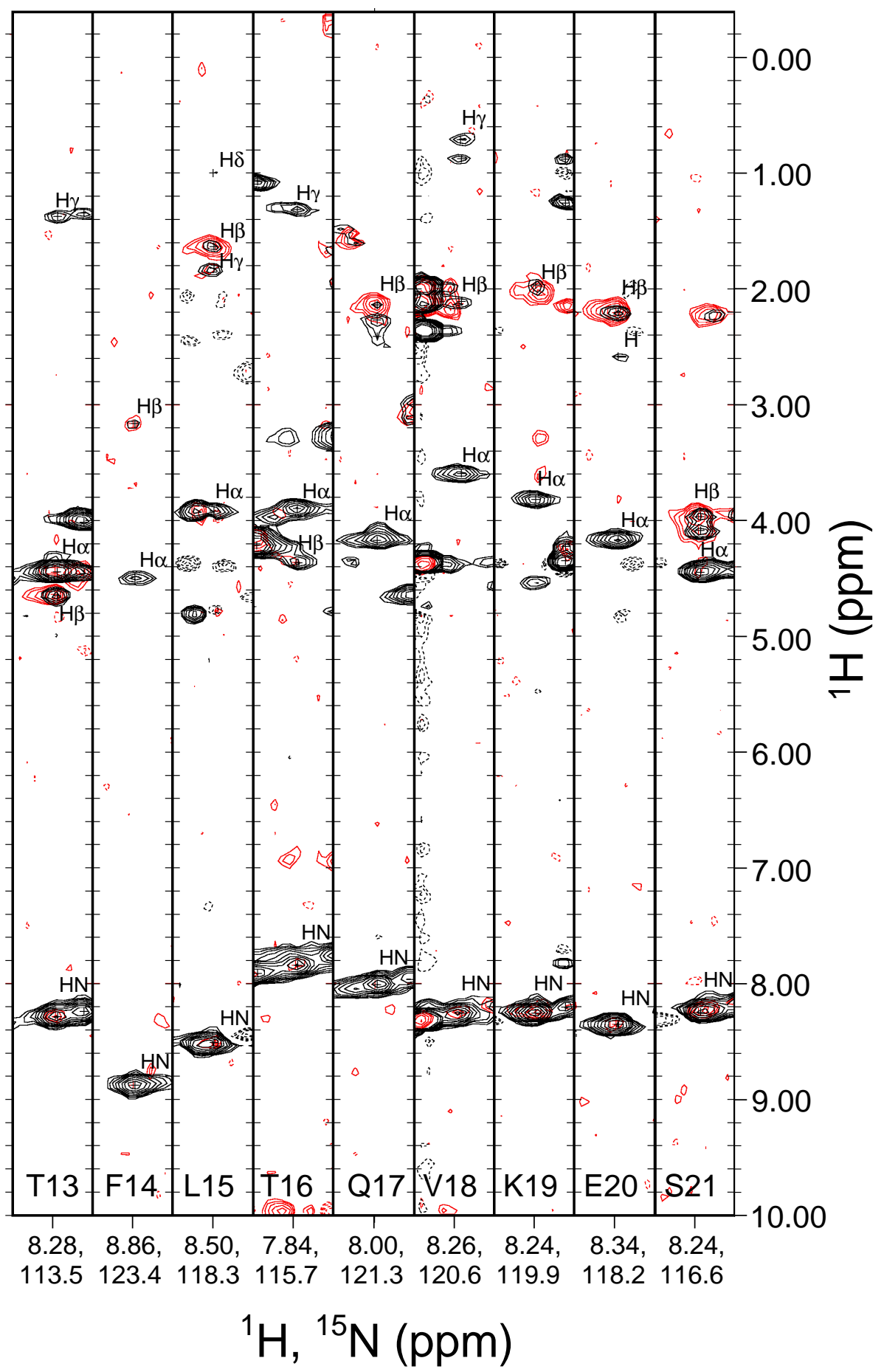

Figure S1: Strips, corresponding to residues $13-21$, of the ${ }^{15} \mathrm{~N}-$ TOCSY-HSQC (black contours) and the HNHB (red contours). Assignments of backbone and sidechain peaks are labelled. Each strip has a width of $0.15 \mathrm{ppm}$ in ${ }^{1} \mathrm{H}$ and shows a single ${ }^{15} \mathrm{~N}$ plane, centred at the chemical shifts shown. 